\title{
SHOMAS: Intelligent Guidance and Suggestions in Shopping Centres
}

\author{
Javier Bajo ${ }^{1}$, Juan M. Corchado ${ }^{1}$, Yanira De Paz ${ }^{1}$, Juan F. De Paz ${ }^{1}$, Sara Rodríguez ${ }^{1}$, Quintín \\ Martín $^{1}$ and Ajith Abraham ${ }^{2}$ \\ ${ }^{1}$ Departamento Informática y Automática Universidad de Salamanca \\ Plaza de la Merced s/n, 37008, Salamanca, Spain \\ \{ jbajope; corchado; yanira; fcofds; srg; qmm\}@usal.es \\ ${ }^{2}$ School of Computer Science, Yonsei University, Seoul, South Korea \\ Email: ajito.abraham@ieee.org
}

\begin{abstract}
This paper presents the SHOMAS multiagent system that provides guidance on leisure facilities and suggestions for shopping in malls. The multiagent architecture incorporates reactive and deliberative agents that take decisions automatically. This paper presents a deliberative agent that provides suggestions in execution time, with the help of case-based planners. This agent is described together with its guidance and suggestion mechanism. SHOMAS has been tested successfully, and the results obtained are presented in this paper.
\end{abstract}

Keywords: multiagent system; case-based reasoning; RFID; Shopping multiagent system (SHOMAS)

\section{Introduction}

This paper presents the SHOMAS Multiagent System, developed for guiding and advising users in Shopping Centres (also known as shopping malls). A shopping centre is a dynamic environment, in which shops change, promotions appear and disappear continuously, etc. The proposed system helps users to identify a shopping or leisure plan as well as to identify other users within a given shopping mall. Multiagent systems (MAS) are specifically recommended for solving dynamic distributed systems. In this particular case SHOMAS users require a wireless device (mobile or PDA) to download their own agent and to interact with the multiagent system.

This distributed system uses Radio Frequency Identification (RFID) [35] technology to ascertain users' location in order to provide security and to optimize their time in the mall. The motivation for the development of such a multiagent system derives from one of the more distinctive characteristics of shopping malls, namely their dynamism, in the sense that in many cases the users are interested in a 
particular product and do not know where to buy it or have very little time, so that new shops or promotions need to be advertised to shoppers. SHOMAS also helps users to identify or locate other users and provides the personnel of the centre with updated information. From the user's point of view the complexity of the solution has been reduced with the help of friendly user interfaces and a robust and easy to use multiagent system.

The proposed reactive user agents in SHOMAS interact directly with a deliberative planning CBP agent which uses a case-based reasoning (CBR) [1], [20] architecture, that allows the agent to make use of past experiences to find the best plans to achieve goals. This particular agent uses a Case-Base Planning (CBP) system, specifically designed for plan construction. CBP agent is a deliberative agent that works at a high level with the concepts of Believe, Desire, Intention (BDI) [8], [9]. The CBP agent has learning and adaptation capabilities, which facilitate its work in dynamic environments. A CBP agent is therefore a particular type of CBR-BDI agent [13], which uses case-based reasoning as a reasoning mechanism, which allows it to learn from initial knowledge, to interact autonomously with the environment as well as with users and other agents within the system, and to have a large capacity for adaptation to the needs of its surroundings. The CBP Agents calculate the most re-plan-able intention (MRPI), which is the plan than can be easterly substituted by other in case the initial plan gets interrupted (for example if the user of the system decides to stay longer in a shop or to interrupt the plan to take a coffee). This agent follows the 4 stages of a CBR system, during the retrieval stage, it selects the previous plans (from the plan/case-base) with information that can be reused to satisfy the problem restriction. During the adaptation stage the agent creates a vectorial hyper dimensional space that includes all the restriction using a B-splines technique. The geodesic plans (curves in the vectorial hyper dimensional space) are identified using the Euler Theorem. Such geodesic plans guaranty minimum risk and constant efficiency, as it will be demonstrated in section 3, and are possible plans that satisfy the agent objectives. The geodesic plan with the Minimum Jacobi Field will be the most replan-able intention. The Minimum Jacobi Field guaranties that it is the plan with more density of other plans around it, and therefore, in case the plan gets interrupted, a closer plan will be easily identified to 
carry out the agent objectives. During the revision stage the user confirms the plan and during the retain stage the plans are stored depending on their efficiency.

Then, this paper presents a distributed architecture and a deliberative CBP guiding agent, for wireless environments. The proposal presented has been used to develop an open wireless system, which is capable of incorporating agents that can provide useful guidance and advice services to the users not only in a shopping centre, but also in any other similar environment such as the labour market, educational system, medical care, etc. Users (clients in the mall) are able to gain access to information on shops and sales and on leisure time activities (entertainment, events, attractions, etc) by using their mobile phone or PDA. Mechanisms for route planning when a user wants to spend time in the mall are also available. Moreover, it provides a tool for advertising personalized offers (a shop owner will be able to publicise his offers to the shopping mall users), and a communication system between management, the commercial sector or shoppers.

SHOMAS has been tested in the Tormes Mall in Salamanca (Spain) with interesting results. The system performance has been positive, after a period of technical adaptation, the user response has also been positive, and some aspects of the mall's management have improved substantially. The shops owners were the most reticent to using the system for several reasons as explained in the conclusions.

Section two presents the problem which motivates most of this research and a state of the art of the existing works. Section three describes the SHOMAS wireless multiagent system, then section four introduces the CBP Agent and finally, the system is evaluated and the conclusions discussed.

\section{Related Work}

The Mall has become one of the most prevalent alternatives to traditional shopping. A shopping centre is a cluster of independent shops, planned and developed by one or several entities, with a common objective. The size, commercial mixture, common services and complementary activities developed are all in keeping with their surroundings. Every shopping mall has a permanent image and a certain common management. A shopping mall needs to be managed and, the management includes resolving incidents or problems in a dynamic environment. As such, a shopping mall can be seen as a 
large dynamic problem, whose administration depends on the variability of the products, users, opinions, etc [7]. The continuous growth of the Internet and the unstoppable advance of technology implies the need to change in the commercial strategies. Between these new strategies it is worth mentioning the development of different E-Commerce systems [18], [23], [11], [34]. E-Commerce allows users to shop through the Internet, receive personalized promotions or request guidance. The incorporation of artificial intelligence techniques has led to further studies and to the modelling of the mall problem in terms of agents and multi-agent systems [30], [15], [21], [28]. These authors focus on the shopping problem and on the suggestions that can be made to users. The growing use of handheld devices in recent years has led to new necessities as well as to a great opportunity to extend traditional commerce techniques and apply new techniques. These new devices facilitate the use of new interaction techniques, for instance, some systems focus on meeting scheduling decision support systems [24], and remark the importance of ontology model definition in these domains [25]. Another systems focus on facilitating users with guidance or location systems [2], [37], [39] by means of their wireless devices. Bohnenberger et al. [6], present a decision-theoretic location-aware shopping guide in a shopping mall as a kind of virtual shop assistant. Bohnenberger et al. [7] propose the use of decision-theoretic planning, but their system can't provide the option of replanning in execution time. Ontology definition is important in the development of decision support systems for route planning. Some authors, as Belmonte et al. [4] propose ontologies for multiagent systems for the bus fleet management domain. SHOMAS uses the CBP mechanism for replanning in execution time and incorporates RFID technology to automatically assess a user's location. Furthermore SHOMAS uses past experiences to take new decisions, which increases the personalization and adaptation capabilities of the system as well as the success of the guidance.

\section{SHOMAS Architecture}

The architecture of the SHOMAS multiagent system incorporates "lightweight" agents that can reside in mobile devices, such as phones, PDAs, etc. [7], and therefore support wireless communication (Wi-Fi, Bluetooth) which facilitates the portability to a wide range of devices. These user agents make it possible for a user to interact with the MAS in a very simple way, downloading 
and installing a personal agent in his mobile phone or PDA. The system also incorporates one agent for each shop in the shopping mall. These agents can calculate the optimal promotions (those of greater sales success) and services at a given moment by considering the retails data and the user profiles. The core of the MAS is a guiding agent in charge of the generation of plans (routes) in response to a user's request, looking for the best shopping or leisure time alternatives. The agent has to take into account the user profile, the maximum amount of money that the user wants to spend and the time available. The generation of routes must be independent of the shopping mall management, in the sense that it is not appropriate to use the same knowledge base (or all the knowledge) controlled by the managemnet. Only the knowledge corresponding to the offers and promotions at the moment of guidance should be used. Otherwise the user will be directed to the objectives of the shopping mall management. As can be seen in Figure 1 there are three types of agents in SHOMAS: the CBP guiding agent, Shop agents situated in each shop and User agents situated in the user mobile device. Each User agent communicates to the nearest shops and can communicate to the CBP agent. Shop agents communicate to CBP agent and User agents.

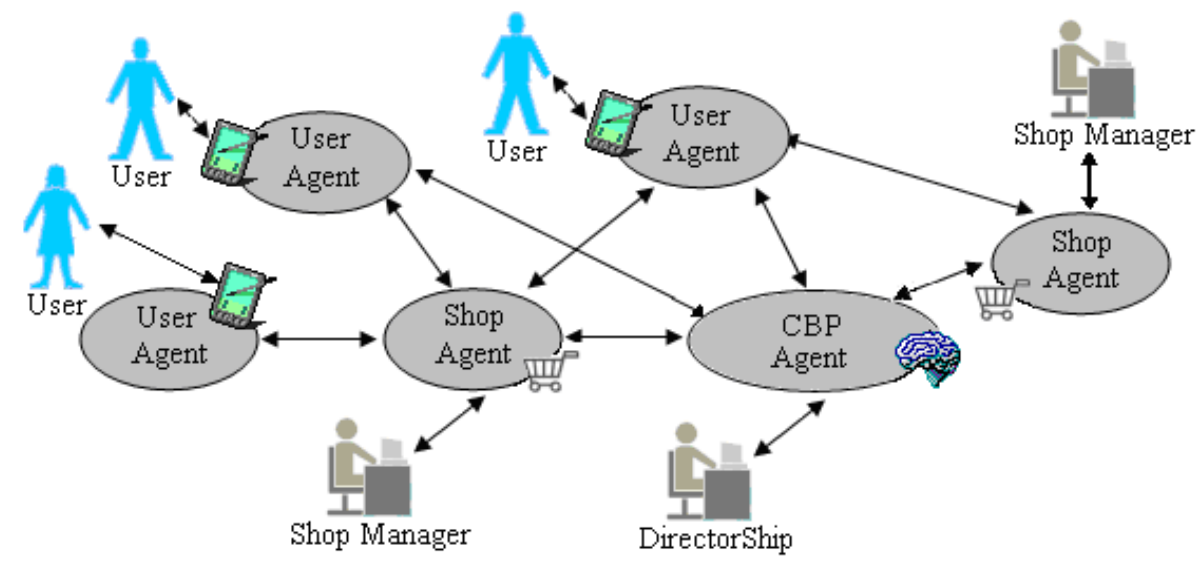

Figure 1. SHOMAS: CBP agent, Shop agents and User agents.

One of the major problems in the development of an architecture based on multiagent system is that there are currently no clear standards or well developed methodologies for defining the steps of analysis and design that need to be taken. There are at present a number of methodologies: Gaia [38], AUML [13], INGENIAS [31], TROPOS [19], MESSAGE [16]. The option chosen to define an appropriate analysis and design methodology for the problem to be resolved is one that combines Gaia 
[38] and (Agent UML) AUML [13], [29], in an attempt to take advantage of both [2]. Through the Gaia analysis, two models are obtained: the role model and the interaction model. The roles identified in the system are Communicator, Finder, Profile Manager, Store Operator, Promotions Manager, Clients Manger, Analyst, Incidents Manager and Planner. These roles will be explained in detail in the following paragraphs. For each of these roles, it will be necessary to specify its particular attributes: responsibilities, permission, activities and protocols [38].

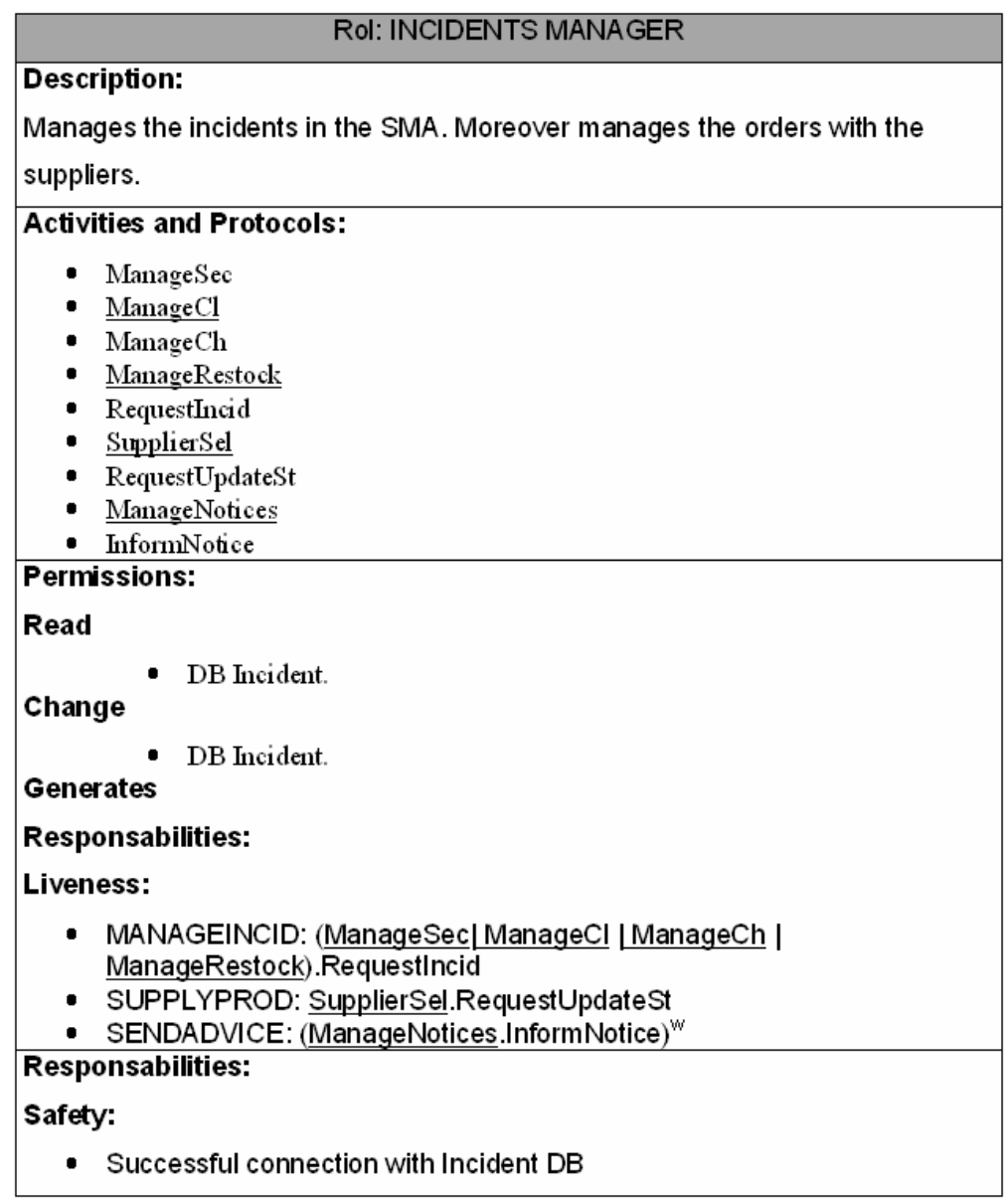

Figure 2. Gaia Incidents Manager role model for the shopping mall problem.

As an example, we shall present the Incidents Manager role: In Figure 2 we can see how the Incidents Manager role is responsible for managing incidents, managing supply events and managing the suggestion system. The protocols used (presented in section 2.1) are those requesting the execution of an action to solve an incident, requesting the update of the store and sending suggestions. The actions that are carried out manage different types of incidents (security, client lost, restock or charge), select the most suitable supplier and managing news or suggestions. The role must have permission to 
access and update the incident database. Its liveness responsibilities are as follows: MANAGEINCID which continually solves incidents; SUPPLYPROD responsible for looking for the best supplier; SENDADVICE, which makes it possible to manage the suggestions and news system. Lastly, the safety responsibilities that the Incidents Manger role has are those which can establish a valid connection with the incident database.

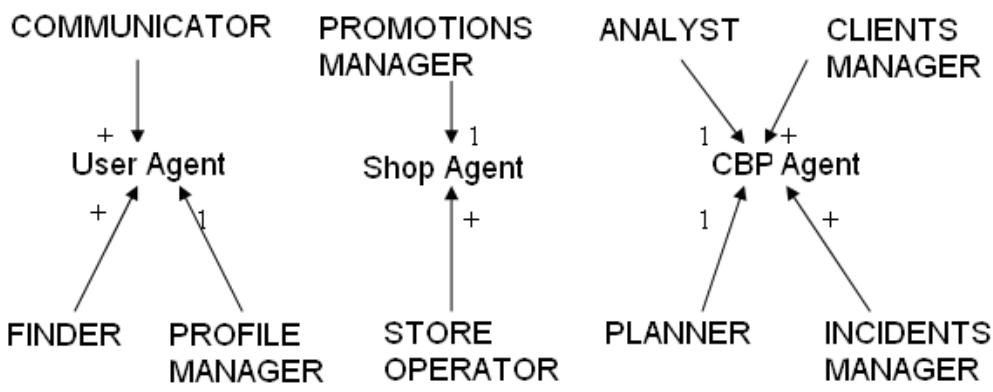

Figure 3. Gaia agent model for the shopping mall problem.

Once the Gaia analysis has been finalised, the Gaia design is carried out. In the Gaia design process three models are considered: agent model, services model and acquaintance model [38]. As can be seen in Figure 3, the agent model shows the types of agents that appear in the system, and the number of instances for each agent type that can be executed in execution time. For example the Shop agent plays the Promotions Manager and Store Operator roles.

After applying Gaia, the result consists of a high-level abstraction design. At this point the Gaia design is transformed so that AUML techniques can be applied. The AUML design provides class diagrams for each agent, collaboration or sequence diagrams for each interaction, state, and activity diagrams to represent internal states and protocol diagrams to model communicative acts [13], [29]. Studying the requirements of the problem, three agent types have been chosen:

- The User Agent plays three roles, the Communicator role manages all the communications of a user; the Finder role looks for devices nearby, trying to identify other users with similar preferences or locate a given user (in this case the use of RFID technology is fundamental); finally the Profile Manager role obtains a user profile.

- The Shop agent plays two roles, the Store Operator is in charge of managing the store (operations on stored products database), and, moreover, monitors product shortages, in order to prevent 
under-supply; and the Promotions Manager role controls the retails in each shop, as well as the promotions that every shop is offering to its clients.

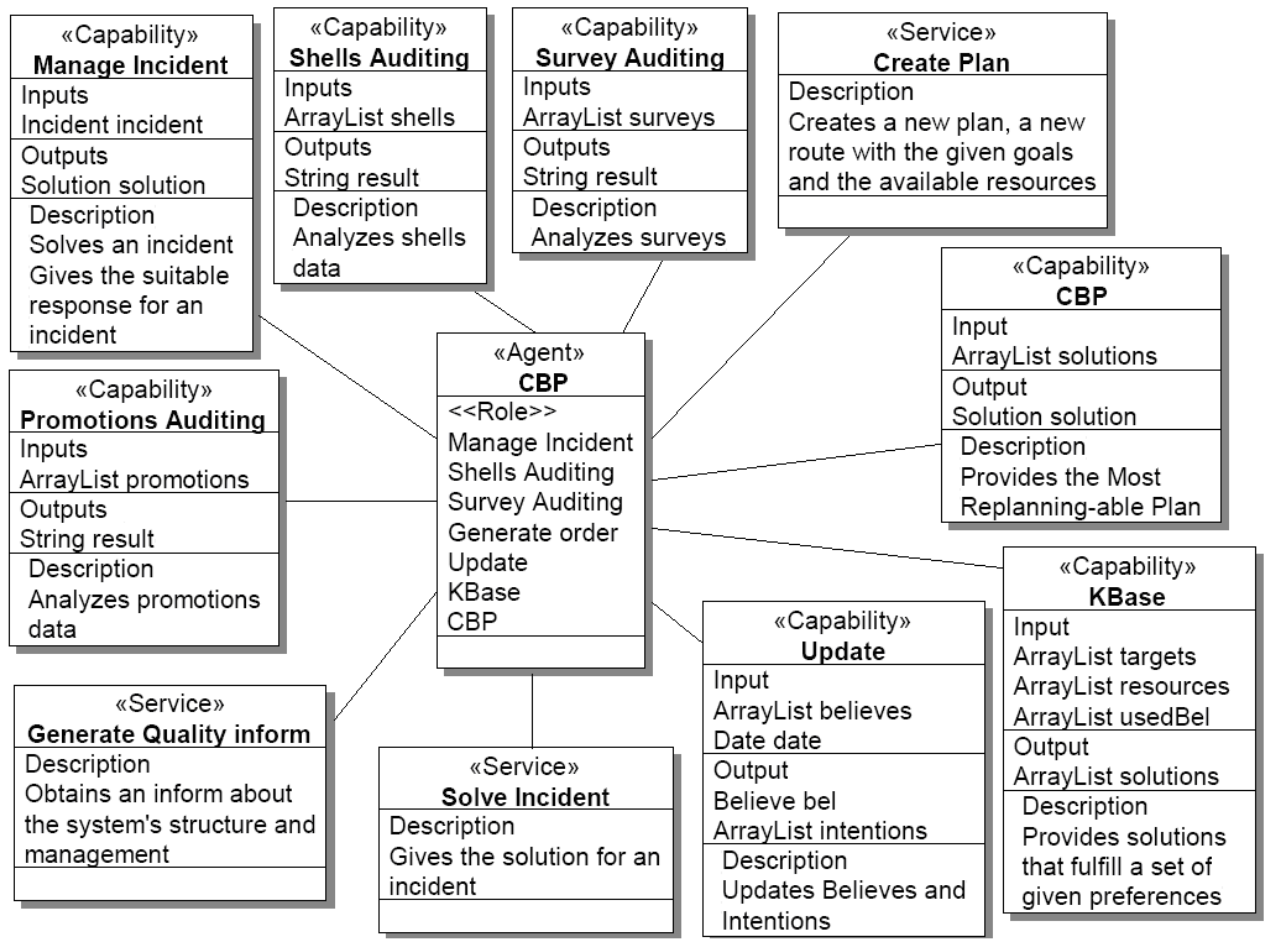

Figure 4. CBP agent class diagram.

- The CBP guiding agent plays four Gaia roles which are divided into seven AUML capabilities: the Clients Manager role deals with the management of user profiles and controls the connected users at any given moment; the Analyst role carries out periodic evaluations on retails, promotions and surveys data trying to provide a good quality of service; the Incidents Manager role manages incidents in the mall, such as sending suggestions, or solving a wide range of problems (security, alerts, lost children, etc.); the Planner role is the most important role in our system. The Planner creates a route identifying the most suitable shops, promotions or events to the user profile and available resources at one particular moment. As can be seen in Figure 4, the Planner role is implemented through three AUML capabilities (Update, KBase and CBP), that make up the Casebased planning cycle explained in detail in Section 3 of this paper. The use of RFID technology allows the CBP agent to locate persons in the mall for security or strategic reasons. Where there is a safety concern - as with young children or the elderly, for example - microchips or tags can be used (Sokymat ID Band Unique Q5 with a chip Hitag S 256) mounted on bracelets worn on the 
wrist or ankle [35]. These chips or transponders use a $125 \mathrm{kHz}$ signal. The door readers (Hitag HT RM401 and mobile WorkAbout Pro RFID) sensors [35], are installed in strategic areas within the mall. Each reader sends a pulse of radio energy to the tags and listen for the tag's response. The signal received from a tag is sent to the CBP agent in order to be processed.

\subsection{SHOMAS in Operation}

As far as the interaction in SHOMAS is concerned, the Gaia interaction model describes the dependencies and relations between roles. Each interaction in which roles are involved requires protocols. In the MAS presented in this paper the following protocols have been considered: RequestPromotionsData when the CBP or user (through his User agent) ask about promotions data and a Shop agent sends the response, RequestProductState when the CBP agent asks for the situation of any products. SolveConsult when a user in the mall interacts with the User agent and makes a query to a Shop agent and receives the response, AlertShortage for a Shop agent to inform the CBP agent about a product shortage, InformOrderSupplier for a Shop agent to inform the CBP agent about an order being carried out, InformProductsState when a Shop agent informs the CBP agent about the state of its products; InformPromotionsState for a shop to send periodic information about promotions to the CBP agent, SolveIncident for a Shop or User agent to indicate to the CBP agent that an incident has occurred and to receive the response, SolveGuidance (includes the AUML RequestSuggestion, InformSuggestion, RequestSugg, RequestReSug, OptimalPlan, InformReplan, InformSugg, InformReSugg, SimilarCases and OptimalSolution) when a user requests guidance from the User agent and the User agent asks the CBP agent about a plan and receives the response; finally, Notify is used for the CBP agent to send suggestions to User or Shop agents. For example, when an user asks for a new route, the User agent uses the SolveGuidance protocol. The CBP agent sends the guidance and keeps receiving the results of each of the subgoals proposed (each of the intermediate states proposed in the plan). If necessary a re-planning will be made.

The interactions between the agents in SHOMAS and the interactions of the mall users with SHOMAS are described in detail by means of AUML protocol diagrams and AUML interaction 
diagrams. The AUML protocol diagrams are directly obtained from the Gaia protocols explained above, and show the interaction between the multi-agent system agents as well as the different roles that can be taken up by the different agents and the interactions between these roles. It is habitual to use a collaboration diagram [13], [29]. Due to the amplitude of the collaboration diagrams, in this paper we only show an example of collaboration diagram in SHOMAS.Figure 5 shows an interaction initiated by a user in order to request a suggestion. The user communicates directly to his personal User agent indicating the new case description. The User agent communicates to the CBP agent that executes the Update role to retrieve information about similar cases solved in the past. The Update role may need to consult the one or more Shop agents to assess its information. Once the similar cases have been retrieved, the CBP agent changes to the KBase role and obtains the optimal solution (plan) for the current case. The CBP agent changes to the CBP role and revises the proposed solution. Finally the CBP agent gives the plan to the User agent and the User agent communicates the optimal plan to the user. The user can change his preferences or opinions at execution time, so a replanning may be necessary. The user indicates the new preferences to his User agent. The User agent communicates to the $\mathrm{CBP}$ agent that plays the $\mathrm{CBP}$ role. The $\mathrm{CBP}$ role may need to consult one or more Shop agents at the moment of replanning. Once the CBP agent obtains the new optimal solution (replan), the user is notified through his personal User agent. The CBP agent studies the user's preferences and complaints.

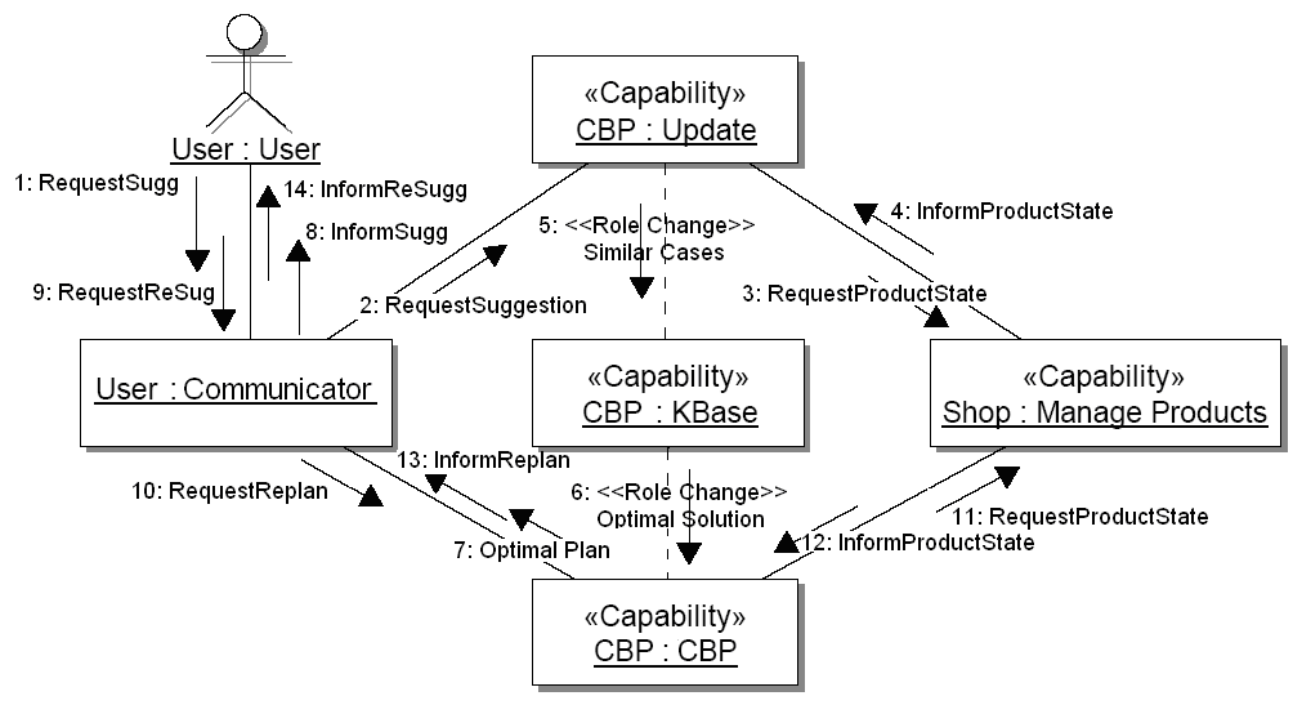


Figure 5. Request suggestion AUML collaboration diagram.

The interactions have been implemented using a robust wireless LAN. In SHOMAS Wi-Fi and Bluetooth devices coexist together with RFID devices. A secure and authenticated access to the data is provided. The use of different authorisations for users, logins and passwords, and the encryption of messages using a public key infrastructure and SSL (Secure Socket Layer) have already been implemented. Moreover, the RFID tag only contains the user's identification number, and not personal data. The communication mechanism is provided by the Jade platform [5]. In this way, both ontologies and languages used are those proposed by the Foundation for Intelligent Physical Agents. Jade uses the Agent Communication Language (ACL) defined by the Foundation for Intelligent Physical Agents. The agents send and receive java objects that represent ACL messages in accordance with a series of protocols. The majority of protocols appear in the libraries offered by Jade [5]. Furthermore, the Foundation for Intelligent Physical Agents - Semantic Language (FIPA-SL) contents language is used [17].

\subsection{Cases in SHOMAS}

The main concept when working with CBR systems is the concept of case. The case structure for a user profile shown in Table 1, is defined using Case Based Markup Language (CBML) [14]. The structure is defined through feature labels. The items, attributes and their values and weights are labelled. In our problem, three main attributes have been considered: personal data, retail/leisure time data and interest data. The retail/leisure attribute is composed of business type, business identification, product type, product identification, price, units and date attributes. The interest data attribute is composed of retail time and frequency, monthly profit both business and product, extracted from retail data, and the explicit attributes obtained from questionnaires. Each attribute has a value, noun or adjective, and a weight assigned. Since the number and type of business is extensive, they were classified into leisure time (cinema and recreational), catering (restaurant, fast food and pizza) and public retail (clothes, shoes, computing, supermarket and optical). The products have been also 
classified, for example the films are divided in action, comedy, terror and drama. A case is java object, composed of java objects.

TABLE I

USER PROFILE CASE FIELDS

\begin{tabular}{ll}
\hline \hline Case Field & Measurement \\
\hline PERSONALDATA & User Personal Data (UserData) \\
RETAILDATA & Retails (RetailsData) \\
INTEREST & User interests (UserInterest) \\
\hline \hline
\end{tabular}

A case corresponding to a user profile contains the description of the problem (personal data on the user and information about his purchases or attendance at activities within the commercial centre) and the solution to the problem description (interests of the user, tastes and tendencies).

The agent in charge of providing suggestions is a CBP agent and the case structure for guidance is shown in Table 2. The agent deals with multiple objectives, as shown in Figure 4, derived from the task of coordinating all the shops (promotions, incidents, bargains, sales), user management and planning and optimising routes. The routes and promotions proposed to a user take into account the user profile and his resources (money and time) at the moment that the request for guidance is made. It contains a mall map and an estimation of the time employed walking by the user. The CBP agent is able to generate routes, analyze retails and promotion data, manage incidents and manage users at the same time.

TABLE 2

GUIDANCE CASE FIELDS

\begin{tabular}{ll}
\hline \hline Case Field & Measurement \\
\hline USER & User profile (UserProfile) \\
MONEY & Money to spend (Money) \\
TIME & Time (Time) \\
INIT & User initial location (Location) \\
PREF & User preferences (Preference) \\
SOLUTION & Solution and efficiency (Solution) \\
\hline \hline
\end{tabular}

A case corresponding to a guidance contains the description of the problem (user profile, money and time available, user initial location and preferences) and the solution to the problem description (suggestion).

The platform chosen for the implementation was Jadex [32]. The Jadex agents deal with the concepts of beliefs, goals and plans. A belief can be any type of java object and is stored 
in the beliefs base. A goal represents a motivation that has influence on the agent behaviour. A plan is a java procedure and is executed in order to achieve goals. Jadex has the advantage of allowing programmers to include their own deliberative mechanisms. Moreover it offers us all the communication advantages that Jade [5] provides (including the LEAP add-on).

\section{CBP guiding agent}

A CBP agent is a particular type of CBR-BDI agent [13], which uses a CBR system [1], [20] to generate plans from cases. The purpose of a case-based reasoning system is to solve new problems by adapting solutions that have been used to solve similar problems in the past [1]. The deliberative agents, proposed in the framework of this investigation, use this concept to gain autonomy and improve their guiding capabilities. CBP agent is a deliberative agent that works at a high level with the concepts of Believe, Desire, Intention (BDI) [8], [9]. In a CBP agent, each state is considered as a belief and the objective to be reached may also be a belief. The intentions are plans of actions that the agent has to carry out in order to achieve its objectives [9], so an intention is an ordered set of actions; each change from state to state is made after carrying out an action (the agent remembers the action carried out in the past, when it was in a specified state, and the subsequent result). A desire is any of the final states (a place in the shopping centre together with a set of conditions) reached in the past (if the agent has to deal with a situation, which is similar to one in the past, it will try to achieve a similar result to the one previously obtained). This section describes the CBP mechanism integrated within the CBP Agent and the novel planning algorithm proposed for generating routes.

\subsection{Case-based Planning Mechanism}

The CBP agent calculates the most re-plan-able intention (MRPI), which is the plan than can be easterly substituted by other in case the initial plan gets interrupted (for example if the user of the system decides to stay longer in a shop or to interrupt the plan to take a coffee). In a dynamic environment, to have an alternative plan it is important to maintain the efficiency of the system. This 
agent follows the 4 stages of a CBR system (Retrieval, Reuse, Review and Retain). During each reasoning stage the $\mathrm{CBP}$ performs the following actions:

- Retrieval stage. During this step the agent selects the plans, from the plan-base, which are similar or contain similar information to the problem case, that describes the aims of a given shopping mall user and his/her restrictions and user profile.

- Reuse stage. During this stage the agent creates a vectorial hyper dimensional space that includes all the problem restriction (shops, shopping items, open restaurants, films, etc.) using a B-splines technique. Then all possible plans that satisfy a given user requirements are identified. Such plans are the geodesic curves (of the vectorial hyper dimensional space), which can be calculated using the Euler Theorem. Such geodesic plans guaranty minimum risk and constant efficiency, and they satisfy the agent objectives. The geodesic plan with the Minimum Jacobi Field will be the most re-plan-able intention. The Minimum Jacobi Field identifies the plan with more density of other plans around it, and therefore, if the plan gets interrupted, a closer plan will be easily identified to carry out the agent objectives. The CBP agents recommend their users the most re-plan-able intentions.

- Review stage. During the revision stage the user confirms the plan.

- Retain stage: During the retain stage the plans are stored depending on their efficiency as will be shown.

The following sub-section describes this process in detail.

\subsection{Planning Algorithm in a CBP Agent}

The planning algorithm looks for the optimum route, that is, the most re-plan-able route. Let $E=$ $\left\{e_{0}, \ldots, e_{n}\right\}$ the set of the possible interesting places to visit and shop.

$$
a_{j}: \underset{e_{i}}{E} \rightarrow \underset{a_{j}\left(e_{i}\right)=e_{j}}{E}
$$

An Agent plan is the name given to a sequence of actions (1) that, from a current state $e_{0}$, defines the path of states through which the agent passes in order to offer to the user the better path according 
to each user's characteristics. Below, in (2), the dynamic relationship between the behaviour of the agent and the changes in the environment is modelled. The behaviour of agent $\mathrm{A}$ can be represented by its action function $a_{A}(t) \forall t$, defined as a correspondence between one moment in time $t$ and the action selected by the agent, where $\mathrm{T}$ is the set of time and $\mathrm{N}$ is the set of natural numbers.

$$
\text { Agent } A=\left\{a_{A}(t)\right\}_{t \in T \subseteq N}
$$

From the definition of the action function $a_{A}(t)$ a new relationship that collects the idea of an agent's action plan (3) can be defined, where T represents the set of time instants and A represents the set of actions undertaken by the "agent A", the x represents the cartesian product between the sets $\mathrm{T}$ and $\mathrm{A}$. Given a fixed time instant $t$, the "agent A" takes an action "a" that depends on the prefixed time instant. Depending on the action "a", the plan P would be generated for the "agent A".

$$
p_{A}: \underset{\left(t, a_{A}(t)\right)}{\operatorname{TxA}} \underset{p_{A}(t)}{A}
$$

in the following way,

$$
p_{A}\left(t_{n}\right)=\sum_{i=1}^{n} a_{i A}\left(t_{i}-t_{i-1}\right)
$$

Given the dynamic character that we want to print onto our agent, the continuous extension of the previous expression (4) is proposed as a definition of the agent plan, in other words (5) -

$$
p_{A}\left(t_{n}\right)=\int_{t_{0}}^{t_{n}} a_{A}(t) d t
$$

The variation of the agent plan $p_{A}(t)$ will be provoked essentially by: the changes that occur in the environment and that force the initial plan to be modified, and the knowledge from the success and failure of the plans that were used in the past, and which are favoured or punished via learning. $O$ indicates the objectives of the agent and $O^{\prime}$ are the results achieved by the plan. $R$ is the total resources and $R^{\prime}$ represents the resources consumed by the agent. The efficiency of the plan (6) is the relationship between the objectives attained and the resources consumed 


$$
E_{f f}=\frac{\#\left(O^{\prime} \cap O\right)}{\# R^{\prime}}
$$

Where \# means cardinal of a set. The objective is to introduce an architecture for a planning agent that behaves - and selects its actions - by considering the possibility that the changes in the environment block the plans in progress. Given an initial point $e_{0}$ and a final state $\mathrm{e}^{*}$, the term planning problem is used to describe the search for a way to reach a final point $e_{i} \equiv e^{*} \epsilon E$ that meets a series of requirements. Given a problem $E$ and a plan $p(t)$ the functions $O b$ and $R c$ accumulated are constructed from the objectives and costs of the plan (7). For all time points $t_{i}$ there are two associated variables:

$$
O b\left(t_{i}\right)=\int_{a}^{t_{i}} O(t) d t \quad R c\left(t_{i}\right)=\int_{a}^{t_{i}} R(t) d t
$$

This allows us to construct a space representing the environment for planning problems as a vectorial hyper dimensional space where each axis represents the accumulative variable associated with each objective and resource. The planning space, defined in this way, conforms to the following properties:

Property 1: The representations of the plans within the planning space are always monotonously growing functions. This property is obtained because $O b(t)$ and $R c(t)$ are functions defined as positive, function $p(t)$ expressed at these coordinates is constant or growing.

Property 2: In the planning space, the straight lines represent plans of constant efficiency. This property is obtained because if the representations of the plans are straight lines, the slope of the function is constant, and coincides with the definition of the efficiency of the plan, as shown in (8).

$$
\frac{d}{d t} p(t)=c t e \Leftrightarrow \lim _{\Delta \rightarrow 0} \frac{\Delta O(t)}{\Delta R(t)}=c t e
$$

In an n-dimensional space, the extension of the straight concept line is called a geodesic curve. In this sense, the notion of geodesic plans can be introduced, defined as those that maintain efficiency at a constant throughout their development. This way, only the plans of constant efficiency (geodesic 
plans) are considered, because they are the ones of minimum risk. In an environment that changes unpredictably, to consider any different plan to the geodesic plan means to accept a certain risk. The agent must search for the plan that determines a solution with a series of restrictions (that depend on the objectives and resources) $F(O ; R)=0$. In the plans base the plans sought are those that are initially compatible with the problem faced by the agent, with the requirements imposed on the solution according to the desires and in the current state [1]. If all the possible plans $\left\{p_{1}, \ldots, p_{n}\right\}$ are represented within the planning space, a subset of states that the agent has already attained in the past will be obtained in order to resolve similar problems. With the mesh of points obtained (generally irregular) within the planning space and using interpolation techniques, a working hyperplan $h(x)$ can be obtained. The hyperplan encapsulates the information on the set of restrictions from restored experiences, which, by definition verify that $h\left(x_{j}\right)=p_{j} j=1, \ldots, n$ and the planning space is the dimension n). From this hyperplan, geodesic plans can be calculated and the variation calculation is then applied. Suppose, for simplicity's sake, a planning space of dimension 3 with coordinates $\left\{O, R_{l}, R_{2}\right\}$. Between the point $e_{0}$ and the objective points $f_{s} f=\left\{e_{1}, \ldots, e_{m}\right\}$ ( $f_{s} f$ is the set of objective points) and over the interpolation surface $h(x)$, the Euler Theorem [20] guarantees that the expression of the geodesic plans will be obtained by resolving the system of equations in (9), where $\mathrm{R}_{\mathrm{i}}$ is the function accumulated $R, O$ is the function of accumulated $O$ and $L$ is the distance function on the hyperplan $h(x), L=\int_{h} d l$.

In order to obtain all the geodesic plans that, on the surface $h(x)$ and beginning at $e_{0}$, allows us to reach any of the points $e^{*} \in f_{s} f$, a condition must be imposed on the surroundings: the initial point will be $e_{0}=\left(O_{0}, R_{0}\right)$. Once an efficient plan is developed, the plan around it (along its trajectory) is used to create a denser distribution of geodesic plans. The tool that allows us to determine this is called the minimum Jacobi field associated with the solution set. Let $g_{0}:[0,1] \rightarrow S$ be a geodesic over a surface $S$. Let $h:[0,1] x[-\varepsilon, \varepsilon] \rightarrow S$ be a variation of $g_{0}$ so that for each $t \in(-\varepsilon, \varepsilon)$, the set $\left\{h_{t}(s)\right\}_{t \in(-\varepsilon, \varepsilon)}: h_{t}(s)$ for all $t \in(-\varepsilon, \varepsilon)$ are geodesic in $S$ and they begin at $g_{0}(0)$, in other words, they conform to $h_{t}(0)=g_{0}(0)$ for all $t \in(-\varepsilon, \varepsilon)$. Under these conditions, taking the variations to a differential limit (10). 


$$
\begin{gathered}
\left\{\begin{array}{l}
\frac{\partial L}{\partial R_{1}}-\frac{d}{d O} \frac{\partial L}{\partial R_{1}^{\prime}}=0 \\
\frac{\partial L}{\partial R_{2}}-\frac{d}{d O} \frac{\partial L}{\partial R_{2}^{\prime}}=0
\end{array}\right. \\
\lim _{t \rightarrow 0}\left\{h_{t}(s)=g_{0}(s+t)\right\}=\lim _{t \rightarrow 0}\{h(s, t)\}=\left.\frac{\partial g_{0}}{\partial t}\right|_{(s, 0)}=\frac{d g_{0}}{d s} \equiv J_{g_{0}}(s)
\end{gathered}
$$

The term $J_{g 0}(s)$ is given to the Jacobi Field of the geodesic $g_{0}$ for the set $\left\{g_{n}(x)\right\}_{n \in N}$, and in the same way as the definition has been constructed, it is possible to give a measurement for the distribution of the other geodesics of $\left\{g_{n}(x)\right\}_{n \in N}$ around $g_{0}$ throughout the trajectory. Given a set of geodesics, some of them are always $\mathrm{g} *$ that, within their environment, have a greater distribution than other geodesics in a neighbouring environment. This is equivalent to saying that it presents a variation in the distribution of geodesics lower than the others and therefore the Jacobi Field associated with $\left\{g_{n}(x)\right\}_{n \in N}$ reaches its lowest value at $\mathrm{J}_{\mathrm{g}^{*}}$. Let's return to the CBP agent problem that, following the recuperation and variation calculation phase, contains a set of geodesic plans $\left\{p_{1}, \ldots, p_{n}\right\}$. If the $p^{*}$ is selected with a minimum Jacobi Field value, it can be guaranteed that in the event of interruption it will have around it a greater number of geodesic plans in order to continue. This suggests that given a problem with certain restrictions $F(O ; R)=0$, the geodesic plan $p^{*}$ with minimum associated Jacobi field associated with the set $\left\{g_{n}(x)\right\}_{n \in N}$ is called the most re-plan-able solution. The behaviour model $G$ for the CBP agent is (11).

$$
G\left(e_{0}, p_{1}, \cdots, p_{n}\right)=p^{*} \Leftrightarrow \exists n \in N / J_{g_{n}} \equiv J_{g^{*}}=\operatorname{Min}_{n \in N} J_{g_{n}}
$$

If the plan $p^{*}$ is not interrupted, the agent will reach a desired state $e_{j} \equiv e^{*} C f_{s} f, j \epsilon\{1, \ldots, m\}$. In the learning phase, a weighting $w_{f}(p)$ is stored, based on the efficiency obtained for the plan. With the updating of weighting $w_{f}\left(p^{*}\right)$, the planning cycle of the CBP motor is completed. In Figure 2, it is possible to see what happens if $p^{*}$ is interrupted. Let's suppose that the agent has initiated a plan $p^{*}$ but at a moment $t>t_{0}$, the plan is interrupted due to a change in the environment. The geodesic planning meets the conditions of the Bellman Principle of Optimality [30], in other words, each one of the plan's parts is partially geodesic between the selected points. This guarantees that if $g_{0}$ is geodesic 
for interrupted $e_{0}$ in $t_{1}$, because $e_{0}$ changes to $e_{1}$, and $g_{1}$ is geodesic to $e_{1}$ that is begun in the state where $g_{0}$ has been interrupted, it follows that: $g=g_{0}+g_{1}$ is geodesic to $e=e_{0}\left(t_{1}-t_{0}\right)+e_{1}\left(t_{2}-t_{1}\right)$

The dynamic process follows the CBP cycle recurrently: each time a plan finds itself interrupted, it generates from the state reached so far, the surroundings of the plans from the case base and adjusts them to the new problem. With this it calculates the geodesic plans and selects the one which meets the minimum conditions of the associated Jacobi field. In this way, the dynamic planning model of the agent $G(t)$ is characterised as shown in Figure 6. A minimum global Jacobi field $J(t)$ also meets Bellman's conditions of optimality [30], in other words, a minimum global Jacobi field, must select minimum Jacobi fields “in pieces” (12).
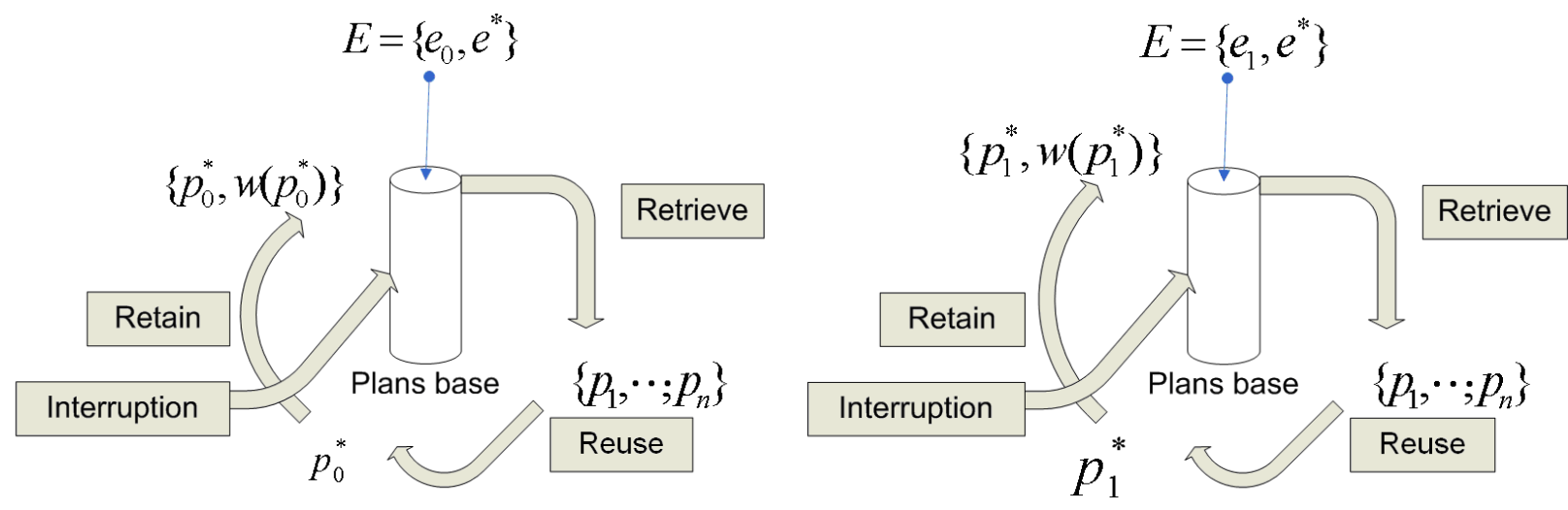

Figure 6. Model for behaviour $\mathrm{G}(\mathrm{t})$.

If on the one hand, successive Jacobi fields generate one Jacobi field, and on the other hand, minimum Jacobi fields generate a minimum Jacobi field, the CBP agent that follows a strategy of replanning $G(t)$ as indicated to survive a dynamic environment, generates a global plan $p^{*}(t)$ that, faced with all possible global plans $\left\{p_{n}(t)\right\}_{n \in N}$, presents a minimum value in its Jacobi field $J_{g^{*}}(t) \equiv$ $J_{p^{*}}(t)$. As such, an agent has been formally defined which in a dynamic environment seeks plans that lend it greater capacity for replanning.

$$
J_{\min }(t)=\left\{J_{\min }\left(t_{1}-t_{0}\right), J_{\min }\left(t_{2}-t_{1}\right), \cdots, J_{\min }\left(t_{n}-t_{n-1}\right)\right\}
$$




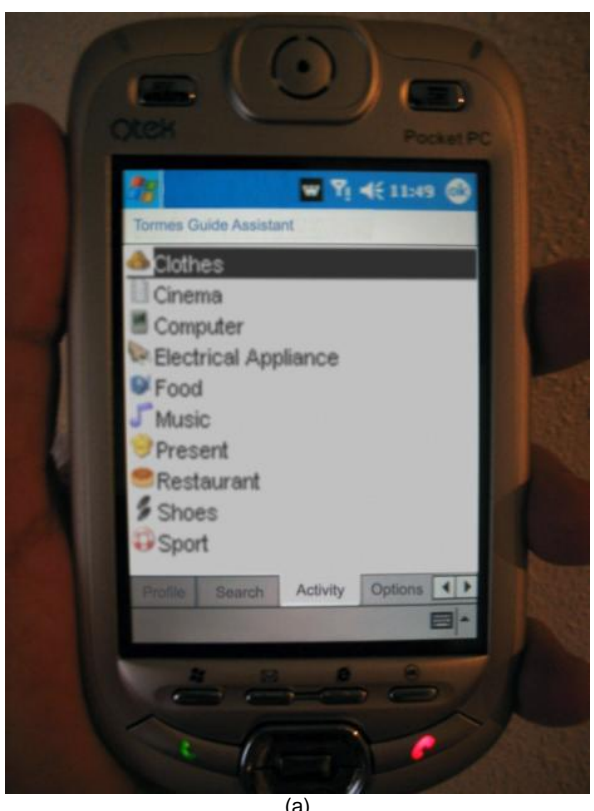

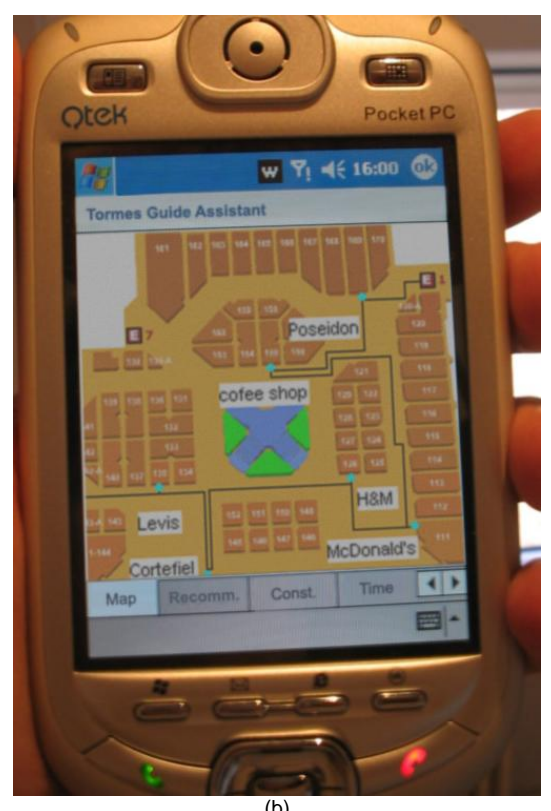

(b)

Figure 7. Screen shots of a user introducing his preferences and the route suggested to the him.

Figure 7 shows a simple example: The mall's main entrance has been taken as the origin of coordinates. Different positions (user, shops, leisure areas) are represented by means of coordinates on a plane $\left(\mathrm{R}^{2}\right)$. Taking into account the user's interests, places to visit are selected and the routes that include those points are traced, and the more easily replan-able route in the event of interruption of the initial plans is proposed; this is done bearing in mind the time available, and the shopping and leisure activities schedule. The chosen route has around itself the largest density of alternative routes, to ensure the success of the proposed plan.

\section{Results and conclusions}

The system described in this paper was tested at the Tormes Shopping Mall in the city of Salamanca during 2005 and 2006. This Shopping centre has 86 different businesses including shops, restaurants, cafes, cinemas, hairdressers and a day nursery. The multiagent system prototype has been tuned and updated during this period and the initial results have been very successful from the technical and scientific point of view. The construction of the distributed prototype has been relatively easy using previously developed CBR-BDI libraries [3], especially since the Mall has a Wi-Fi network and has provided the businesses with Bluetooth and RFID technology. AUML [13], [29] and Gaia [38] provide 
an adequate framework for the analysis and design of distributed agent based systems. The formalism defined in [2], [20] facilitates the straight mapping between the agent definition and the CBR construction. Figure 7 presents two screen shots of the User agent. Figure 7 (a) illustrates a form for introducing information on preferences while the route generated for a user trying to buy clothes and to see an action movie is shown in Figure 7 (b). The security problem in data transmissions (data privacy) was tackled by using the FIPA https protocol [17] and by using a private network to connect Shop agents with the CBP guiding agent.

Figure 8 depicts the interface that the User agent makes available to the user for them to interact with the multiagent system. Figure 8(a) demonstrates how a user may configure his user agent in such a way that he/she will be informed when a user with a certain profile is connected to the system (users have to previously give their consent or activate this kind of service). If users are near each other the Bluetooth protocol facilitates direct user identification, otherwise it has to be done using the Bluetooth, WI-FI or RFID networks of the Shopping Centre. On the other hand, Figure 8(b) illustrates the form that the User agent receives in order to obtain the personal data of the user so that his profile can be created. Finally, in Figure 8(c) we can see the guidance given to a user in which a particular plan is suggested according to their characteristics and preferences, while Figure 8(d) illustrates the result obtained from the suggested plan. In this case the restrictions are the users time and the money to spend. The agents calculate its aims, also, taking into consideration the users preferences and the visit motivation

The fundamental concept when working with a CBR system is the concept of case, so it is necessary to establish a case definition. A case managed by the CBP agent, is composed of the attributes described in Table 2: the user profile - the money available to spend, the time available, the user's initial location, the user's preferences when guidance is given through the PDA interface between the options shown and the solution proposed; The guidance and the result obtained for the guidance success, failed, user opinions, sales results. Cases can be manipulated manually or automatically by the agent during its revision stage, when the user evaluation obtained through questionnaires is given to the system. The agent plans can be generated using different strategies since the agent integrates 
different algorithms. The metrics mechanisms proposed in [10] facilitate the retrieval stage, but the products base and the promotions base must be defined and sorted including metrics that allow it to find similitude, such as the time expected to spend buying each product. The user profile is obtained from retail data and periodic questionnaires.
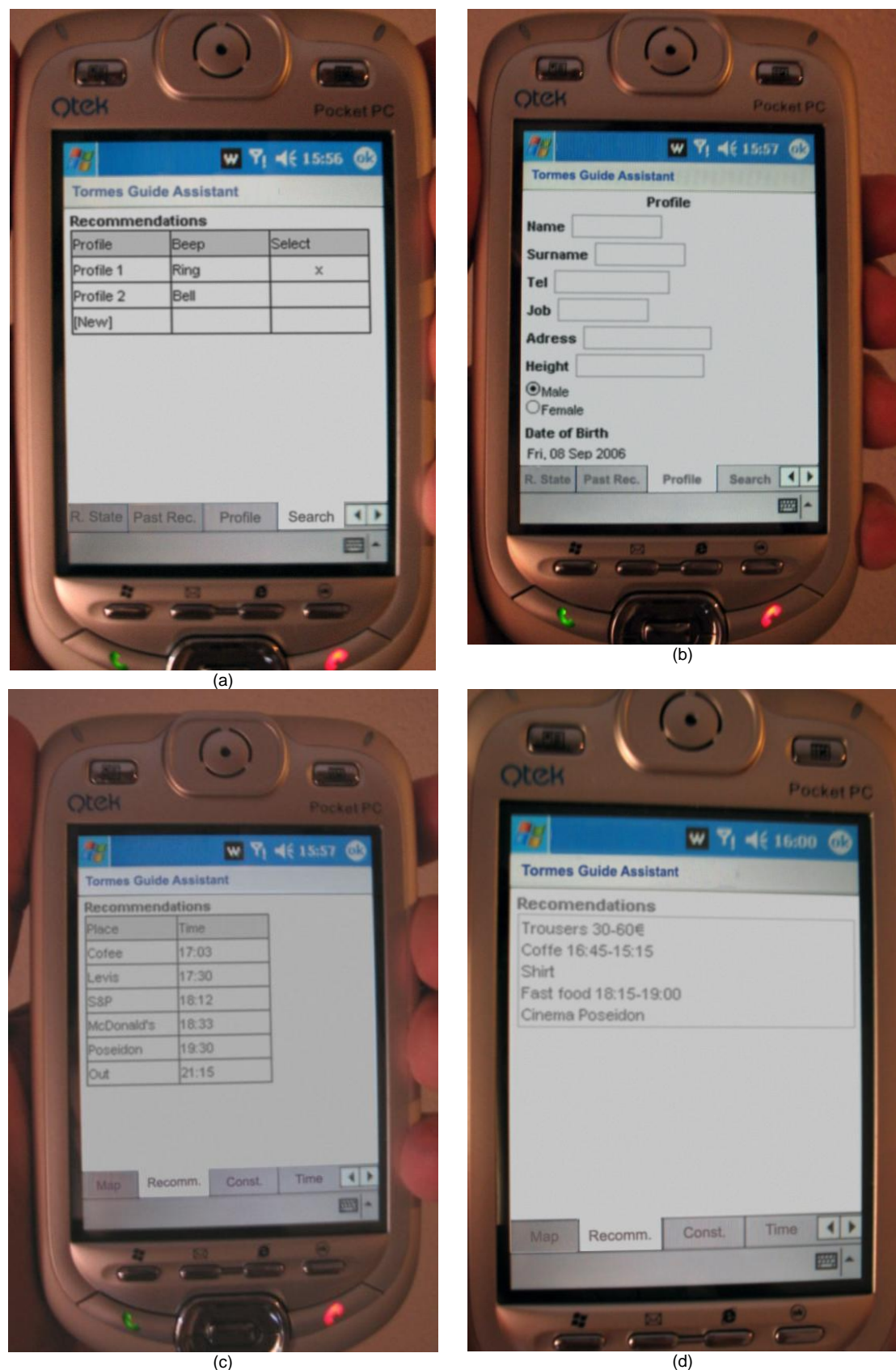

Figure 8. User Agent Screenshots.

The system was tested between September 2005 and December 2006 and obtained promising results. The e-commerce techniques have facilitated user motivation since a user can easily find the 
products he/she is interested in, spend their leisure time more effectively and contact other users with whom he/she can share hobbies or opinions. So the degree of user satisfaction has been improved as observed in the surveys.

The first autonomous prototype was implemented in October 2005 with a test set of 30 users; presently there are over a 2400 different users. The users were selected among users with specific models of terminals supporting the application (they use their own Wi-Fi, Bluetooth devices). The results obtained show that the greater part of users, nearly 67\%, were people between 16 and 30 years old, while the percentage of people older than 40 is less than $3 \%$. However there were no significant differences with respect to user gender. Figure 9(a) shows the users satisfaction level over time, which increased substantially, especially after a second prototype was launched in February 2006, that was more consistent and containing more information about promotions and special offers. At the beginning, the system obtained a low evaluation, basically due to the fact that the system was new and had some bugs; but as cases were incorporated, the products being promoted became closer to the user profile. The user satisfaction is measured from user opinions and by indirect observation on the sales results. The user opinions are obtained from a questionnaire that the user completes every month. Moreover, every time the system provides guidance, it asks the user about his/her degree of satisfaction.

Figure 9(b) depicts the evolution of sales (in thousands of Euros) for the set of special offers, used as a reference over the years to evaluate the evolution of purchases at the commercial centre. They represent a set of 23 promotions that gradually diminish over time. From this data we can see that, comparing the sales of each promotion over the time that the prototype was introduced with the sales from the previous year (within the same time period), the percentage of sales slightly increased. The promotion of these products through the guiding system helped to change tendencies. Figure 9(c) illustrates how the implantation of the guiding system has increased during months 4-6 when traditionally, in this period the number of visitors actually decreased. 

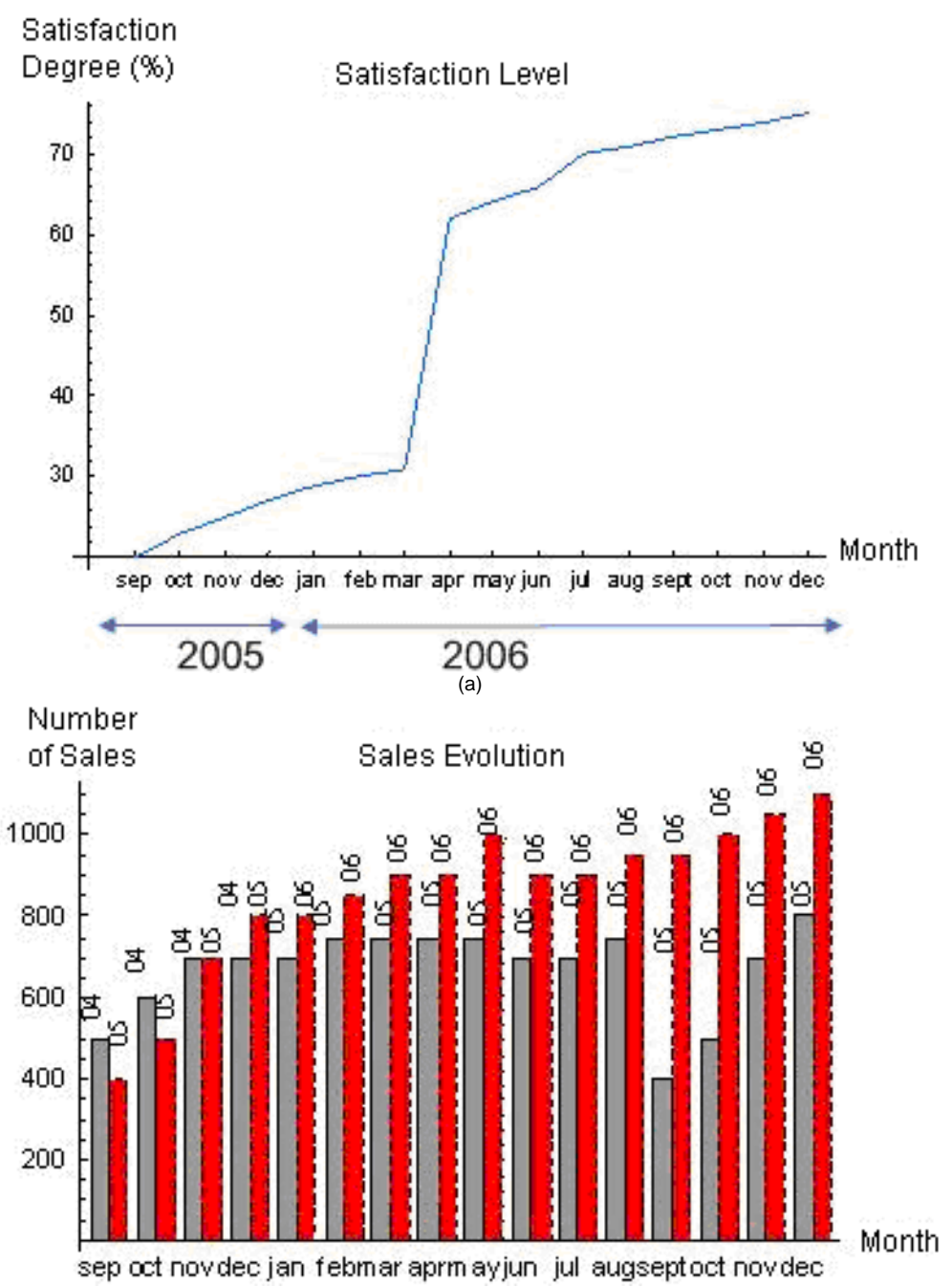

(b)

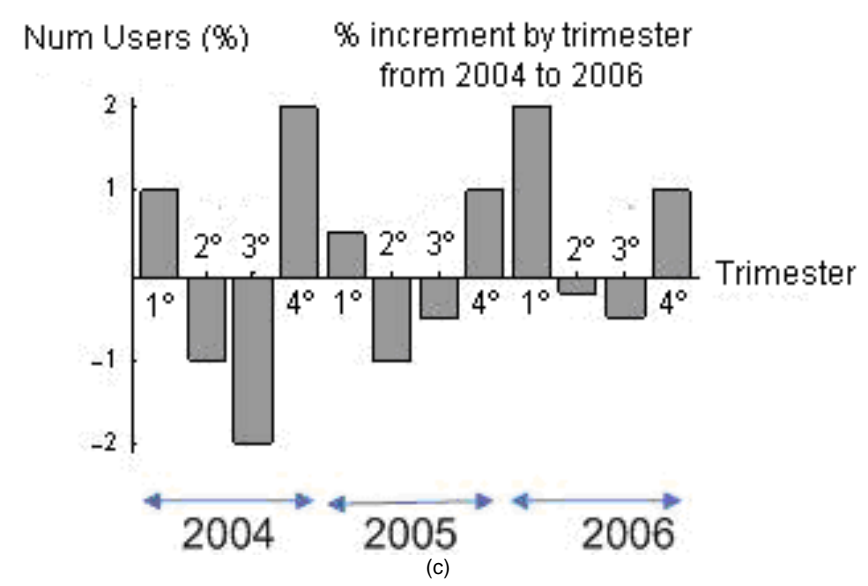

Figure 9. Users satisfaction degree and sales evolution.

Users have noticed the utility of the dynamic replanning, since it is quite usual for them change opinions/objectives in the middle of a plan. CBP is a highly appreciated tool that optimizes the time spent in the shopping mall. Figure 10(a) shows the number of replannings requested per visit among 
visitors who requested guidance. Figure 10(b) shows how the number of plans completed without a request for guidance continually increased because of the capacity of the system to learn and adapt to the interests of the users. This occurred at the same time as the number of users who did not complete the plan requested or that requested modifications to the initial plan (changed the initial objectives) decreased.
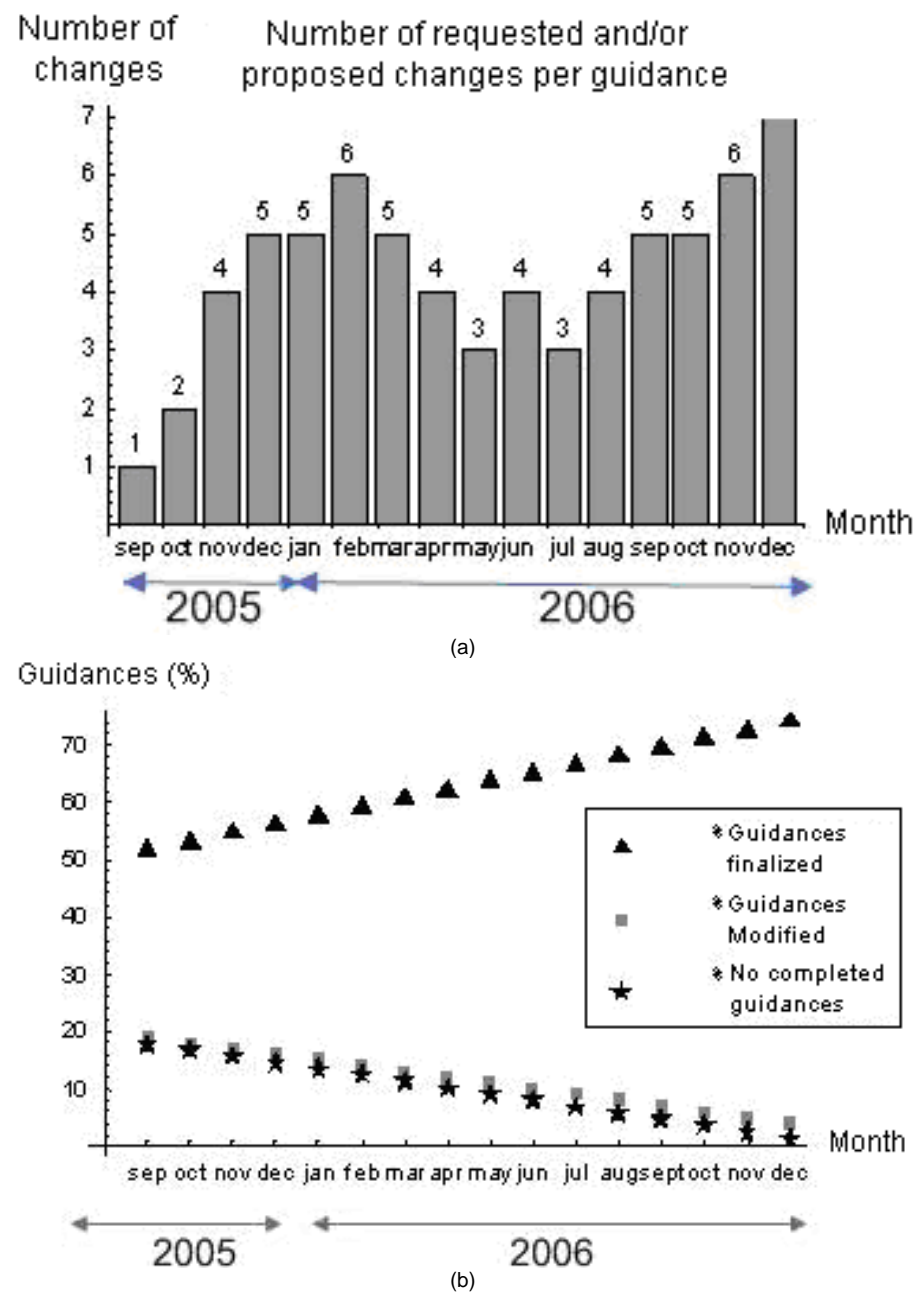

Figure 10. Guidance system evolution.

Figure 11(a) shows the increase in the number of SHOMAS users. In the same way, Figure 11(b) shows how the median number of guidances requested increased during the months between September 2005 and September 2006. A guidance is composed of the problem description (user profile, money, time available, initial location of user and preferences) and the solution (guidance proposed, and result obtained after following up the guidance) given for the problem description given. 


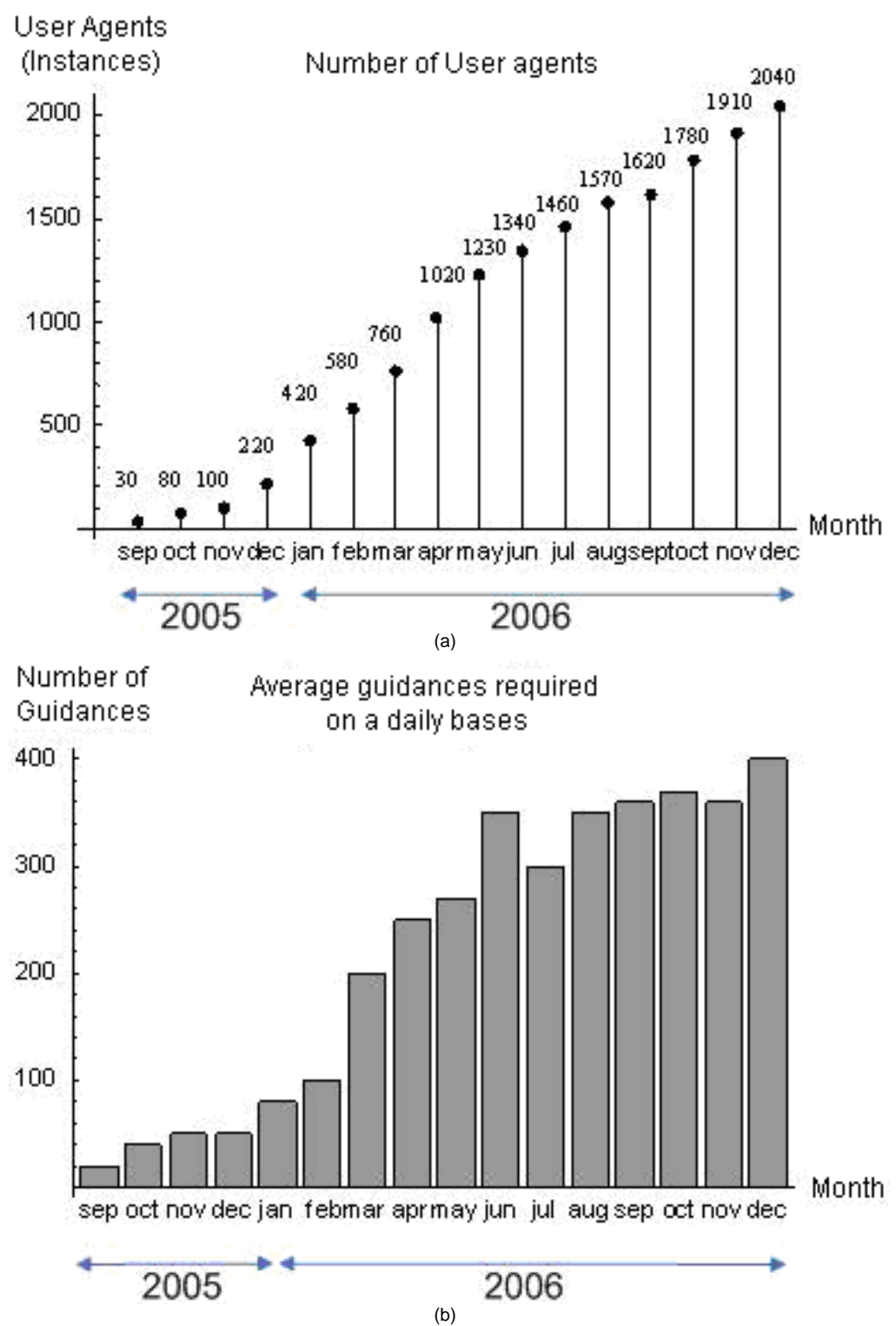

Figure 11. Number of users and guidances evolution

As SHOMAS obtains more information about user profiles, products and habits, the system knowledge increases and the CBP agent provides more optimal plans. The users also need time to get used to the system. The proposed guiding system has been improved to be able to provide adequate guidance in a dynamic way and in execution time. In this sense it is a unique system useful for dynamic environments and open enough to be used in other environments such as health care residences, educational environments or tourist related environments. One of the most demanding services of SHOMAS is the location of a given user by means of the RFID technology or the identification of someone with a given profile, in the same line of web services such as Match.com or 
similar sites. In this sense the system performance has been correct from the logical point of view, although there have been a few technical problems during the initial months of the experiments due, in particular, to the Bluetooth network. This service is used by an average of $46 \%$ of the users. The shop owners are the most reticent about using the guiding system for several reasons: (i) they do not trust the partiality of the guiding systems, since they cannot control whether it is biased or not, (ii) updating the information about products and offers of the shop agents requires specialised human resources and time, since they are not currently integrated with their software packages, (iii) they believe that the CBP agent may favour big shop stores with many offers and (iv) some of them argue that the SHOMAS may confuse some users. Nevertheless most shop managers believe that SHOMAS has more advantages than disadvantages and that the system has helped their businesses attract more customers and, in general, to sell more. They tend to argue that SHOMAS should incorporate a method that guarantees impartiality. The definition of an advanced ontolology can help to achieve this aim and it is necessary to take into account previous works in multi-agent applications as those used in patent document analysis [36], CMMI project monitoring and control [26] or health care [27]. This is our next challenge.

\section{Acknowledgements}

This work has been supported by the MCYT project TIC2003-07369-C02-02 and the JCyL Project JCYL-2002-05-SA104A05. Special thanks to Sokymat for the technology provided.

\section{References}

[1] A. Aamodt and E. Plaza, Case-Based Reasoning: foundational Issues, Methodological Variations, and System Approaches, AI COM. Vol. 7 (1994) 39-59.

[2] J. Bajo, J.F. De Paz, Y. De Paz and J.M. Corchado Integrating Case Planning and RPTW Neuronal Networks to Construct an Intelligent Environment for Health Care. Expert Systems with Applications, 43 (3) In Press 
[3] J. Bajo, J.F. De Paz, D.I. Tapia and J.M. Corchado Distributed Prediction of Carbon Dioxide Exchange Using CBR-BDI Agents. International Journal of Computer Science. INFOCOMP, (2007) 16-25

[4] M.V. Belmonte, J.L. Perez-de-la-Cruz and F. Triguero, (2008). Ontologies and agents for a bus fleet management system. Expert Systems with Applications, 34(2), 1351-1365.

[5] F. Bellifemine, G. Cairea, A. Poggib and G. Rimassac JADE: A software framework for developing multi-agent applications. Lessons learned. Information and Software Technology,50 (1-2) 10-21

[6] T. Bohnenberger, A. Jameson, A. Krüger, A. Butz: Location-aware shopping assistance: Evaluation of a decision-theoretic approach, in: Proceedings Mobile HCI'02, Lecture Notes in Computer Science, Vol. 2411 (Springer, Berlin, 2002) 159-169.

[7] T. Bohnenberger, O. Jacobs and A. Jameson (2005). DTP meets user requirements: Enhancements and studies of an intelligent shopping guide, in: Proc. PERVASIVE-05, Lecture Notes in Computer Science, Vol. 3468 (Springer, Berlin, 2005) 279-296.

[8] M.E. Bratman, Intentions, Plans and Practical Reason (Harvard University Press, Cambridge, M.A., 1987).

[9] M.E. Bratman, D. Israel, and M.E. Pollack, Plans and resource-bounded practical reasoning, Computational Intelligence 4 (1988) 349-355.

[10] R. Burke. Knowledge-based Recommender Systems. Encyclopedia of Library \& Information Systems, 69 (32), (New York, USA, 2000).

[11] K. Chang Leea and N. Chung A web DSS approach to building an intelligent internet shopping mallnext term by integrating virtual reality and avatar. Expert Systems with Applications, 28 (2) (2005) 333-346.

[12] J.M. Corchado, J. Aiken, E.S. Corchado, F. Fdez-Riverola Evaluating the air-sea interactions and fluxes using an instance-based reasoning system AI Communications, 18 (4) 247 -256

[13] J.M. Corchado, R. and Laza, Constructing Deliberative Agents with Case-based Reasoning Technology, International Journal of Intelligent Systems, 18 (2003) 1227-1241. 
[14] L. Coyle, C. Hayes and P. Cunningham Representing Cases for CBR in XML, in Expert Update, 6 (2) (2003) 7-13.

[15] J. Eriksson and N. Finne, MarketSpace: an open agent-based market infrastructure, Uppsala Master's thesis in Computing Science 106, Examensarbete DV3, (1997).

[16] EURESCOM MESSAGE: Methodology for engineering systems of software agents. Technical report P907-TI1, EURESCOM (2001)

[17] FIPA Foundation for Intelligent Physical Agents. http://www.fipa.org (2007).

[18] M. Ga, Agents in E-commerce, Communications of the ACM, 42, No.3 (ACM Press, 1999) 7980.

[19] P. Giorginia, J. Mylopoulosb and R. Sebastiani Goal-oriented requirements analysis and reasoning in the Tropos term methodology. Engineering Applications of Artificial Intelligence, 18 (2) (2005) 159-171

[20] M. Glez-Bedia, J.M. Corchado, E. Corchado and C. Fyfe, Analytical Model for Constructing Deliberative Agents, Engineering Intelligent Systems, 3 (2002) 173-185.

[21] S.P. Fonseca, Martin L. Griss, Reed Letsinger, An Agent Mediated E-Commerce Environment for the Mobile Shopper, Technical Report, HPL-2001-157 Hewlett-Packard Laboratories, 2001.

[22] M.P. Huget Agent UML notation for multiagent system design Internet Computing, IEEE 8 (4) (2004) 63-71.

[23] J. Kim, R. LaRose, Interactive e-commerce: Promoting consumer efficiency or impulsivity?, Journal of Computer-Mediated Communication 10 (2004).

[24] C.S. Lee, C.Y. Pan, An Intelligent Fuzzy Agent for Meeting Scheduling Decision Support System, Fuzzy Sets and Systems..142 (3) (2004) 467-488.

[25] C.S. Lee, C.C.Jiang, and T.C. Hsieh, A genetic fuzzy agent using ontology model for meeting scheduling system, Information Sciences. 176 (9) (2006) 1131-1155. 
[26] C.S. Lee, M.H. Wang, and J.J. Chen, Ontology-based Intelligent Decision Support Agent for CMMI Project Monitoring and Control, International Journal of Approximate Reasoning, 48 (1) $62-76,2008$.

[27] C.S. Lee and M.H. Wang, Ontology-based Intelligent Healthcare Agent and Its Application to Respiratory Waveform Recognition, Expert Systems with Applications. 33. (3) (2007) 606619.

[28] P. Maes, R. Guttman and A. Moukas, Agents That Buy and Sell, Communications of the ACM, 42, (3) (1999) 81-91.

[29] J. Odell, R. Levy, and M. Nodine, FIPA Modeling TC: Agent Class Superstructure Metamodel. FIPA meeting and interim work, 2004.

[30] Z. Pan, B. Xu, H. Yang and M. Zhang Content-based personalised recommendation in virtual shopping environment. International Journal of Business Intelligence and Data Mining, 1 (4) (2006) 430-449

[31] J. Pavón, J. Gómez-Sanza, A. Fernández-Caballerob and J.J. Valencia-Jiménez Development of intelligent multisensor surveillance systems with agents Robotics and Autonomous Systems, 55 (12) (2007) 892-903

[32] A. Pokahr, L. Braubach, and W. Lamersdorf, Jadex, Implementing a BDI-Infrastructure for JADE Agents, in: EXP - In Search of Innovation (Special Issue on JADE), Vol 3, (3) (Telecom Italia Lab, Turin, Italy, September 2003) 76-85.

[33] W.S. Powel, Approximate Dynamic Programming: Solving the Curses of Dimensionality (Wiley Series in Probability and Statistics, Wiley, New York, 2007).

[34] H.H. Shung, Helping Online Customers Decide through Web Personalization, IEEE Intelligent Systems, 17 (6) (2002) 34-43.

[35] Sokymat. http://www.sokymat.com. (2007).

[36] V.W. Soo, S.Y. Lin, S.Y. Yang, S.N. Lin and S.L. Cheing, (2006). A cooperative multi-agent platform for invention based on patent document analysis and ontology. Expert Systems with Applications, 31(4), 766-775 
[37] D.I. Tapia, J.F. De Paz, S. Rodríguez, J. Bajo and J.M. Corchado Multi-Agent System for Security Control on Industrial Environments. International Transactions on System Science and Applications (In Press)

[38] M. Wooldridge and N.R. Jennings and D. Kinny, The Gaia Methodology for Agent-Oriented Analysis and Design, Journal of Autonomous Agents and Multi-Agent Systems, 3 (3). (2000) 285-312.

[39] T. Yoshino, T. Muta, J. Munemori, NAMBA: location-aware collaboration system for shopping and meeting, IEEE Transactions on Consumer Electronics, Vol. 48 (3) (2001) 470-477. 